\title{
Albrecht von Löwenstein, miles sancti sepulchri
}

\author{
Von Folker Reichert
}

In memoriam Maximilian Weltin (1940-2016)

Albrecht, Graf von Löwenstein und Herr zu Scharfeneck, führte ein unstetes Leben. 1536 als vierter Sohn Graf Friedrichs I. geboren, kam er als Regent der seit 1504 zum Herzogtum Württemberg gehörigen Grafschaft Löwenstein nie in Frage. Bei der Teilung des väterlichen Erbes von den Brüdern kurzgehalten, ja unterversorgt, hielt er sich wahrscheinlich an einem fremden Hof auf und unternahm verschiedene Reisen, über deren Verlauf und Umstände allerdings gar nichts bekannt ist. Rom, Paris und London scheint er mit eigenen Augen gesehen zu haben. Später übernahm er Legationen im Auftrag verschiedener Herren und bewährte sich in deren Dienst. Als Kriegsunternehmer kam er zu Geld. Wichtige Kriegsschauplätze lernte er kennen. Als er des Umherziehens müde geworden war, fand er am württembergischen Hof eine feste Dienststellung. Aber immer noch war er viel unterwegs. Denn man schätzte seine Erfahrung in Kriegen und sein Geschick als Gesandter.

Als Albrecht im März 1587 in seinem Löwensteiner Schloss auf den Tod lag, konnte er auf ein Leben zurückblicken, das vom Unterwegssein geprägt war und dem Reisen viel verdankte. Persönliche Erfahrungen und Kenntnisse, monetärer Gewinn, Ruf und Ansehen an verschiedenen Höfen: das alles basierte auf seinen Reisen. Doch die längste, ereignisreichste und wohl auch riskanteste von allen war jene, die ihn als 25-jährigen Mann im Sommer 1561 von Venedig aus ins Heilige Land und ein Jahr später über den Sinai und Ägypten nach Venedig zurück führte.

Hermann Ehmer hat facettenreich und quellengesättigt ein Lebensbild Albrechts gezeichnet, das Standbild eines geharnischten Ritters auf dem Friedhof in Abstatt (Kr. Heilbronn) als einzig erhaltenen Rest seines Grabmals identifiziert und als „Krönung seiner Reisen“ die Heiliglandfahrt gewürdigt ${ }^{1}$. Deren Bedeutung, auch

${ }^{1}$ Hermann Ehmer, Graf Albrecht von Löwenstein (1536-1587). Jerusalempilger und Kriegsunternehmer, Diplomat und Beamter, in: ZWLG 72 (2013) S. 153-226 (Zitat S. 169). $\mathrm{Zu}$ Albrechts Jerusalemreise vgl. Friedrich EmLein, Die Pilgerfahrt des Grafen Albrecht von Löwenstein in das Heilige Land und auf den Berg Sinai in den Jahren 1561/62, in: Jahrbuch des Historischen Vereins „Alt-Wertheim“ 1930, S.72-98; 1931, S. 100-119; 1932, S. $98-121$. 
für den Grafen selbst, wird man aber noch besser verstehen, wenn man sie in die Geschichte der spätmittelalterlich-frühneuzeitlichen Jerusalemreisen einordnet und vor diesem Hintergrund interpretiert ${ }^{2}$. Dazu sollen die folgenden Überlegungen und Hinweise dienen.

\section{Reisen nach Jerusalem im 16. Jahrhundert}

Albrecht von Löwenstein war nicht allein unterwegs, sondern reiste in Gesellschaft. Das darf man wörtlich verstehen. Denn von jeher taten sich die Jerusalempilger zu „Gesellschaften“ zusammen und ließen sich bei der Vorbereitung und Planung ihrer Reise durch ausgewählte Mitglieder vertreten. Diese Gruppen sollten nicht zu groß und nicht zu klein sein und dem Einzelnen für vorübergehende Zeit ein stabiles soziales Umfeld garantieren ${ }^{3}$. Albrecht von Löwenstein trat an die Spitze der „schwäbischen Gesellschaft“, die aus neun Mitgliedern bestand. Zwei weitere Gesellschaften (eine „kaiserliche“, eine „fränkische“) wurden von den übrigen Deutschen gebildet. Wie (und ob) sich die anderen Reisenden organisierten, wissen wir nicht. Am Ende drängten 177 Personen auf das Schiff und nahmen einander den Platz weg - Deutsche, Italiener, Niederländer, Spanier und Franzosen, Pilger und Seeleute, auch einige Frauen (von denen eine schwanger wurde) und ein indianischer Christ, der die Grabeskirche abzeichnete, um nach deren Muster in seiner Heimat ein Kloster zu bauen, dessen Abt er dann sein wollte. Vier von den deutschen Passagieren hielten ihre Eindrücke in schriftlicher Form fest und trugen so zu der seit dem 14. Jahrhundert stetig wachsenden Pilgerliteratur bei:

1. Albrecht von Löwenstein und Scharfeneck;

2. der Straßburger Ritterbürger Jacob Wormbser, der den Nutzen seiner Reise auf die schöne Formel brachte, man könne lernen, dass es nicht überall in der Welt so zugehe „wie am Kochersberg im Elsass“"

3. der oberösterreichisch-kärntnische Adlige Bartlmä (Bartholomäus) Khevenhüller zu Aichelberg (später Freiherr auf Landskron und Wernberg), der während einer Kavalierstour durch Frankreich und Spanien in die Fänge der Inquisition

${ }^{2} \mathrm{Zu}$ den Jerusalemreisen allgemein vgl. Ursula GanZ-BLÄTtLer, Andacht und Abenteuer. Berichte europäischer Jerusalem- und Santiagopilger (1320-1520) (Jakobus-Studien 4), Tübingen 1990; Folker Reichert, Erfahrung der Welt. Reisen und Kulturbegegnung im späten Mittelalter, Stuttgart 2001, S.137ff.; Nicole Chareyron, Pilgrims to Jerusalem in the Middle Ages, New York 2005 (franz. 2000); Colin Morris, The Sepulchre of Christ and the Medieval West. From the Beginning to 1600, Oxford 2005; Sabine Penth, Die Reise nach Jerusalem. Pilgerfahrten ins Heilige Land, Darmstadt 2010.

${ }_{3}^{3}$ Folker Reichert, Die Reise des Pfalzgrafen Ottheinrich zum Heiligen Land 1521, Regensburg 2005, S. 26.

${ }^{4}$ Vgl. Wolfgang Treue, Abenteuer und Anerkennung. Reisende und Gereiste in Spätmittelalter und Früher Neuzeit (1400-1700), Paderborn 2014, S. 166, 333. 
geriet, für den Fall seiner Freilassung eine Wallfahrt nach Jerusalem gelobte und diese dann auch gewissenhaft antrat, freilich ohne dass seine Familie davon wusste $^{5}$;

4. der spanische Obrist und Bürger zu Feldkirch in Vorarlberg David Furtenbach, der aus Überdruss am Kriegswesen den Dienst quittiert hatte und stattdessen eine Wallfahrt unternahm, von der er nicht zurückkehren sollte ${ }^{6}$.

Zwei dieser vier Berichte gingen in Sigmund Feyerabends Sammlung „Reyßbuch deß heyligen Lands“ ein und wurden so schon bald einem breiteren Publikum bekannt, die beiden anderen blieben bis ins 19. bzw. 20. Jahrhundert ungedruckt ${ }^{7}$. Vier Selbstzeugnisse, die über ein und dieselbe Reise berichten, stellen weder einen einmaligen noch einen alltäglichen Fall dar. Pilgerfahrten in den Jahren 1483 (u.a. Felix Fabri aus Ulm), 1493 (u.a. Herzog Friedrich der Weise von Sachsen), 1519 und 1521 (u. a. Pfalzgraf Ottheinrich) werden durch mindestens ebenso viele Texte dokumentiert ${ }^{8}$. Man spricht von „Parallelberichten“ und weiß durch Arnold Esch, was sich alles damit anfangen lässt: Man kann die Texte miteinander vergleichen, um deren Gemeinsamkeiten von den Unterschieden abzugrenzen. Oder man kann die Autoren geradezu gegeneinander treiben, um deren jeweilige Eigenheiten zu erfassen. Der eine reagiert auf die gleiche Situation so, der andere anders. Der Vergleich von Parallelberichten erweist sich somit als eine „Versuchsanordnung,

${ }^{5}$ Ebd., S.60f.; Hans KhevenhüLler, Geheimes Tagebuch 1548-1605, hg. von Georg Khevenhüller-Metsch, bearb. von Günther Probszt-Ohstorff, Graz 1971, S. 10 f.

${ }^{6}$ Reinhold RöHricht, Bibliotheca geographica Palaestinae. Chronologisches Verzeichnis der von 333 bis 1878 verfassten Literatur über das Heilige Land mit dem Versuch einer Kartographie [1890]. Verbesserte und vermehrte Neuausgabe von David H. K. Amiran, Jerusalem 1963, S. 196 ff.; Ders., Deutsche Pilgerreisen nach dem Heiligen Lande, Innsbruck 1900, S. 233 ff., 239 ff.; Stéphane Yerasimos, Les voyageurs dans l'empire ottoman (XIVe $\mathrm{XVI}^{\mathrm{e}}$ siècles), Ankara 1991, S. 253, 256 ff.; Hans-Gert Roloff (Hg.), Die Deutsche Literatur. Biographisches und bibliographisches Lexikon, Reihe II, Bd.2, Bern/Berlin 1991, S. 50-53 (Albrecht von Löwenstein); Ralf C. Müller, Prosopographie der Reisenden und Migranten ins Osmanische Reich (1396-1611) (Berichterstatter aus dem Heiligen Römischen Reich, außer burgundische Gebiete und Reichsromania), Leipzig 2006, Bd.2, S.452ff.; Bd.4, S. 412 ff.; Bd. 5, S. $220 \mathrm{ff}$.; Bd. 10, S. $223 \mathrm{ff}$.

7 Sigmund Feyerabend (Hg.), Reyßbuch deß heyligen Lands / Das ist Ein gründtliche beschreibung aller vnd jeder Meer und Bilgerfahrten zum heyligen Lande, Frankfurt a.M. 1584, fol.188 $-212^{\mathrm{v}}$ (Löwenstein); fol.213 ${ }^{\mathrm{r}}-235^{\mathrm{r}}$ (Wormbser); Ferdinand KHull, Aus dem Tagebuche des Grafen Bartlmä Khevenhüller-Frankenberg, in: Carinthia 86 (1896) S. 73 - 85, 107-114, 129-155, hier S. 129 ff.; Andreas Ulmer, Das Tagebuch des David von Furtenbach aus Feldkirch über seine Hl. Land-Fahrt 1561, in: Alemannia 7 (1933) S. 39-53.

${ }^{8}$ Vgl. die Nachweise bei: Werner Paravicini (Hg.), Europäische Reiseberichte des späten Mittelalters. Eine analytische Bibliographie, Bd.1: Deutsche Reiseberichte, bearb. von Christian Halm, Frankfurt a.M. ${ }^{2} 2001$; ferner Arnold Esch, Vier Schweizer Parallelberichte von einer Jerusalem-Fahrt im Jahre 1519, in: DERs., Alltag der Entscheidung. Beiträge zur Geschichte der Schweiz an der Wende vom Mittelalter zur Neuzeit, Bern 1998, S. 355-399; Reichert, Die Reise des Pfalzgrafen Ottheinrich (wie Anm.3). 
die Individualität freisetzt" ${ }^{\text {" }}$. Das von Esch angeregte Verfahren hat sich mittlerweile vielfach bewährt und wurde auch schon auf die Reise von 1561 angewandt. Der Graf von Löwenstein erwies sich dabei als abenteuerlustig, wenn nicht tollkühn, während Jacob Wormbser eher zur Vorsicht neigte und die ganze Reise am Ende nur noch „langweilig und widerwärtig“ fand ${ }^{10}$. Albrecht dagegen interessierte sich für Mumien, Brutöfen und Giraffen, suchte nach Erklärungen für die Dinge, die ihm seltsam erschienen, besichtigte die Pyramiden von Gizeh von außen und innen und wäre sogar nach Mekka weitergereist, wenn es nicht verboten gewesen wäre ${ }^{11}$. Gut verstand er sich mit dem fast gleichaltrigen Bartlmä Khevenhüller. Gemeinsam ließen sie sich zum Ritter schlagen und assistierten einander dabei. Allerdings unterschieden sich die Motive ihrer Reisen. Kamen bei Löwenstein Frömmigkeit und Abenteuerlust zusammen, so ergab sich Khevenhüllers Heiliglandreise aus einer Notlage. Von sich aus hätte er die Wallfahrt wohl nicht unternommen. Mit dem Besuch des Heiligen Grabs hatte er seine Schuldigkeit getan, den Ritterschlag nahm er mit. Dass auch der Sohn des Schiffsherrn, also ein Bürgerlicher, zum Grabesritter promoviert wurde, hat ihn empört: der "Geiz“ (also das Geld) regiere die Welt, selbst ehrenvolle Auszeichnungen kämen dadurch in Verruf ${ }^{12}$. Sicher wusste er sich auch hier mit dem Grafen von Löwenstein einig.

Man kann aber auch die günstige Quellenlage dazu nutzen, um sich Gedanken über den Verlauf der Heiliglandfahrten nach 1520 zu machen. Man geht gemeinhin davon aus, dass sie - durch den Einfluss der Reformation einerseits, durch die osmanische Expansion im östlichen Mittelmeer andererseits - deutlich reduziert wurden. Sie kamen jedoch nie völlig zum Erliegen. Luthers ausgesprochene Skepsis (Gott frage nach dem Heiligen Grab in Jerusalem so viel „wie nach allen Kühen in der Schweiz“13) spielte zunächst keine Rolle. Auch Protestanten fuhren zum Heiligen Grab (Bartlmä Khevenhüller und Jacob Wormbser zum Beispiel; bei Albrecht selbst ergibt sich ein unklares Bild). Der Untergang des Mamlukensultanats in Ägypten hatte für die christlichen Pilger völlig neue, weniger berechenbare Verhältnisse geschaffen. Mit den Osmanen, den neuen Herren im Heiligen Land,

9 Arnold Esch, Gemeinsames Erlebnis - individueller Bericht. Vier Parallelberichte aus einer Reisegruppe von Jerusalempilgern 1480, in: Zeitschrift für Historische Forschung 11 (1984) S. 385-416.

10 Wolfgang Treue, Peregrinatio Hierosolymitana. Zwei deutsche Ritter auf der Reise durch Palästina und Ägypten in den Jahren 1561-62, in: Friedhelm Burgard u.a. (Hg.), Liber amicorum necnon et amicarum für Alfred Heit. Beiträge zur mittelalterlichen Geschichte und geschichtlichen Landeskunde (Trierer historische Forschungen, Bd.28), Trier 1996, S. 445-458.

11 Feyerabend (wie Anm.7) fol.200v.

12 Khull (wie Anm.7) S. 144f.; Müller (wie Anm. 6) Bd. 4, S. 413, 421: ... es regiert der Geiz zu sebr in der Welt, dz viel erliche orden in verachtung khumen.

13 D. Martin Luthers Werke. Kritische Gesamtausgabe, Bd.8, Weimar 1889, S.562: Denn nach dem grab, do der herr ynn gelegen hatt, welchs die Sarracen ynne haben, fragt got gleych ßo vill, als nach allen kwen von schweytz. 
war schwerer auszukommen. Hatten jene über fast zwei Jahrhunderte hinweg eine Art pragmatischer Toleranz praktiziert, so standen diese in der Tradition des Kriegertums an der Glaubensgrenze und vertraten einen entschiedeneren Standpunkt im Umgang mit den „Ungläubigen“. Die Eroberung der Inseln Rhodos (1522) und Zypern (1570/1571) beseitigte die letzten christlichen Vorposten im östlichen Mittelmeer, an denen sich die Pilger hatten sicher fühlen können. Für manche von ihnen kam auch der Landweg über Konstantinopel wieder infrage ${ }^{14}$.

Aber gerade die Reise Albrechts von Löwenstein und seiner Reisegefährten zeigt, dass es auch seinerzeit möglich war, einigermaßen sicher und in geordneter Form ins Heilige Land und (immer schon mit mehr Mühe) weiter zum Katharinenkloster auf dem Sinai zu reisen, um dort auch die Zeichen der Katharinenritterschaft zu erwerben. Der Grand Tour der Frühen Neuzeit ließ sich durchaus mit den Ausläufern der spätmittelalterlichen Heidenfahrten, einschließlich der adligen Heiliglandreisen, verbinden ${ }^{15}$. Man muss sich fragen, ob die Abenteuer und Beschwernisse, die die Pilger 1561 erlebten (Albrechts Gefangenschaft in Ägypten und seine zweimalige Verwundung, Furtenbachs Erkrankung und Tod, Khevenhüllers Ausplünderung bei der Abreise von Jaffa), die von jeher üblichen waren oder auf die neuen, schwierigeren Umstände seit ca. 1520 zurückgingen. Immerhin wusste man in Venedig über die Probleme der Pilger Bescheid, und David Ott, Fuggerscher Agent, selbstständiger Kaufmann und Vermittler im Kunsthandel, gab die Nachrichten nach Deutschland weiter. Man machte sich Sorgen, blieb aber optimistisch. An Hylff und Ratt (Hilfe und Rat) werde es schon nicht mangeln ${ }^{16}$. Eine Geschichte der Heiliglandfahrten im 16. und 17. Jahrhundert ist ein Forschungsdesiderat. Zweifellos würde die Reise Albrechts von Löwenstein und seiner Mitreisenden eine prominente Rolle in ihr spielen.

${ }^{14} \mathrm{Vgl}$. Alexander Schunka, Die Konfessionalisierung der Osmanen. Protestantische Berichte über den Orient im ausgehenden 16. Jahrhundert, in: Markus FrIEDRICH/Alexander Schunka (Hg.), Orientbegegnungen deutscher Protestanten in der Frühen Neuzeit = Zeitsprünge 16, 1/2 (2012) S. 8-46, hier S. 14.

15 Vgl. Werner Paravicini, Grand Tour. Adeliges Reisen und europäische Kultur vom 14. bis zum 18. Jahrhundert (Beihefte der Francia, Bd.60), Ostfildern 2005.

16 StA Wertheim, R Lit. D Nr. 788; vgl. Ehmer (wie Anm.1) S.174. David Ott (15071579) hatte auch schon den Vertrag der Pilger mit dem Schiffspatron bezeugt (RöHRICHT, Deutsche Pilgerreisen [wie Anm.6] S. 234); zu ihm vgl. Sibylle Backmann, Kunstagenten oder Kaufleute? Die Firma Ott im Kunsthandel zwischen Oberdeutschland und Venedig (1550-1650), in: Klaus Bergdolt/Jochen BrüNING (Hg.), Kunst und ihre Auftraggeber im 16. Jahrhundert. Venedig und Augsburg im Vergleich (Colloquia Augustana, Bd.5), Berlin 1997, S.175-197, hier S.182 ff.; Oswald Bauer, Zeitungen vor der Zeitung. Die Fuggerzeitungen (1568-1605) und das frühmoderne Nachrichtensystem (Colloquia Augustana, Bd.28), Berlin 2011, S. 90, Anm. 191. 


\section{II. Überlieferung und Textgestalt von Albrechts Pilgerbericht}

Albrechts Text wird durch mehrere Handschriften überliefert:

- Augsburg, Universitätsbibliothek, Cod. I.3.4 $13^{17}$

- Berlin, Staatsbibliothek, Ms. Germ. Quart. $1021^{18}$

- Gotha, Forschungsbibliothek, Chart. B $417^{19}$

- Harvard University, Houghton Library, MS Riant $48^{20}$

- Karlsruhe, Badische Landesbibliothek, Bruchsal $11^{21}$

- Kreuzwertheim/Unterfranken, Schloss, 2 Hss. (A, B) im Besitz der Fürsten von Löwenstein-Wertheim-Freudenberg 22

- München, Bayerische Staatsbibliothek, cgm 300223

- Stuttgart, Württembergische Landesbibliothek, Cod. hist. qu. $81^{24}$

- Weimar, Herzogin-Anna-Amalia-Bibliothek, Q 318

- Weimar, Herzogin-Anna-Amalia-Bibliothek, Fo 318

Die meisten dieser Handschriften enthalten ausschließlich Albrechts Pilgerbericht. Dieser sprach für sich und musste nicht mit anderen Reisetexten kombiniert, kommentiert oder illustriert werden, schon gar nicht im Verständnis des Verfassers. Mehrere Handschriften sind auf den 1. Januar 1580 datiert und wurden - zusammen mit einem Neujahrsgruß - an hoch- und zugleich nahestehende Empfänger verschickt. Ein Exemplar (das Karlsruher) ging an Herzogin Dorothea Ursula von Württemberg, ein zweites (das Stuttgarter) an Graf Friedrich von Mömpelgard, künftigen Herzog von Württemberg, ein drittes an Kurfürst Ludwig VI. von der Pfalz (eine der beiden Handschriften in Kreuzwertheim). Ein viertes lässt sich erschließen. Es war Herzog Ludwig von Württemberg zugeeignet, ist derzeit aber

17 Karin SchneIDER, Deutsche mittelalterliche Handschriften der Universitätsbibliothek Augsburg: Die Signaturengruppen Cod. I. 3 und Cod. III. 1 (Die Handschriften der Universitätsbibliothek Augsburg II 1), Wiesbaden 1988, S. 104.

${ }^{18}$ Hermann Degering, Kurzes Verzeichnis der germanischen Handschriften der Preussischen Staatsbibliothek, Bd.2: Die Handschriften in Quartformat, Leipzig 1926, S. 170 .

19 Freundliche Auskunft von Frau Cornelia Hopf, Gotha.

20 Vgl. L. de Germon/L. Polain, Catalogue de la Bibliothèque de feu M. le Comte Riant, Bd. 2, 1, Paris 1899, S. LIV (seit 1899 im Besitz der Harvard University).

${ }^{21}$ Die Handschriften der Badischen Landesbibliothek in Karlsruhe, Bd.13: Die kleinen Provenienzen, beschrieben von Armin Schlechter/Gerhard Stamm, Wiesbaden 2000, S. $403 \mathrm{f}$.

${ }^{22}$ Mikrofilme im Staatsarchiv Wertheim (StA Wertheim, A 66 Nr. 16). Vgl. Ehmer (wie Anm.1) S.170, Anm. 51.

${ }^{23}$ Die deutschen Handschriften der K. Hof- und Staatsbibliothek in München nach Schmeller's kürzerem Verzeichnis. Erster Theil, München 1866, S. 333.

${ }^{24}$ Die historischen Handschriften der Königlichen öffentlichen Bibliothek zu Stuttgart, beschrieben von Wilhelm von HEYD, Bd.2: Die Handschriften in Quarto und Octavo, Stuttgart 1891, S.32. 
nicht aufzufinden ${ }^{25}$. Bedacht wurden also jene Herrschaften, denen sich Albrecht in besonderem Maße verbunden wusste. Von der Pfalzgrafschaft ging die Herrschaft Scharfeneck, von Württemberg die Grafschaft Löwenstein zu Lehen. Der Herzogsfamilie war Albrecht durch seine Stuttgarter Dienststellung verpflichtet ${ }^{26}$.

Jedes der erhaltenen Widmungsexemplare enthält eine längere Widmungsvorrede, nicht völlig gleichen Inhalts, aber ähnlichen Tenors. Mehrfach sei der Verfasser von hochstehenden Persönlichkeiten aufgefordert worden, die Aufzeichnungen von seiner Heiliglandreise im Druck zu publizieren. Doch obwohl er nur notiert habe, was er mit eigenen Augen gesehen habe und was ihm wirklich widerfahren sei, habe er sich zu einer Drucklegung nicht durchringen können. Man hätte es ihm ja falsch auslegen können, nämlich als Ambition und Fabelei. Vor allem diejenigen, die von der Welt nichts gesehen und ihre Tage lieber inheimisch onnd bey den ibrigen verbracht hatten, könnten versucht sein, seinen Reisebericht als Phantasieprodukt zu betrachten. Um aber seinen guten Willen zu beweisen, habe er die Abschriften anfertigen lassen und an ausgesuchte Adressaten verteilt. Deren Grundlage war: das originall ${ }^{27}$.

Die Vorrede enthält einige Topoi: der Unwille des Autors, sein Werk an die Öffentlichkeit zu geben, das Drängen der Leser, die Versicherung, nur Wahrhaftiges zu berichten, die Berufung auf Augenzeugenschaft und auf das mögliche Zeugnis der Mitpilger. Trotzdem lässt sich ihr entnehmen, dass Albrecht nicht an einer weiteren Verbreitung des Büchleins interessiert war. Vielmehr begnügte er sich mit den wenigen Lesern, von denen er annahm, dass sie verstehen würden, was ihm seine Reise bedeutete. Er bezeichnete seinen Bericht als „Itinerarium“ oder „Reisbuch“, einmal sogar als „Rhapsodie“ (rhapsotia), also als Dichtung ${ }^{28}$, und ging davon aus, dass ein breites Publikum nichts Rechtes damit anfangen konnte. Die schön geschriebene Handschrift galt ihm als Edition. Deren Zirkulation in höfischen Kreisen war ihm Publizität genug ${ }^{29}$.

Trotzdem kam es wenige Jahre später zur gedruckten Publikation. Sie basierte auf einer weiteren Abschrift, die Albrecht seiner Schwägerin Amalia von Baden

${ }^{25}$ Erwähnt in den Widmungsvorreden des Stuttgarter und des Karlsruher Exemplars. RöHRIсHT verzeichnet eine Handschrift im „Königl. Würtemberg. Filialarchiv“ in Ludwigsburg (Bibliotheca [wie Anm.6] S.197). Anfragen in den Staatsarchiven Ludwigsburg und Wertheim führten zu keinem Ergebnis.

26 Vgl. Ehmer (wie Anm.1) S. 167 ff., $199 \mathrm{f}$.

27 Stuttgart, Württembergische Landesbibliothek, Cod. hist. qu. 81, fol. $3^{\mathrm{r}}-4^{\mathrm{r}}$; Karlsruhe, Badische Landesbibliothek, Bruchsal 11, fol. $2^{r-v}$; Kreuzwertheim, Hs. B, fol. $3^{r}-4^{r}$.

${ }^{28}$ Ebd., fol. $3^{\mathrm{v}}$.

${ }^{29}$ Vgl. dazu grundsätzlich Felix HeInzer, Marsilio Ficinos Libellus de comparatione solis ad deum. Von der Dedikationshandschrift für Herzog Eberhard im Bart zum Tübinger Druck von 1547, in: Sabine Holtz/Albert Schirrmeister/Stefan Schlelein (Hg.), Humanisten edieren. Gelehrte Praxis im Südwesten in Renaissance und Gegenwart (VKgL B 196), Stuttgart 2014, S.17-31. 
dediziert hatte ${ }^{30}$. Inwieweit und ob überhaupt der Verfasser in den Vorgang involviert war, wissen wir nicht. Druckort war das „Reyßbuch des heyligen Lands“, das Sigmund Feyerabend 1584 herausbrachte. Der geschäftstüchtige Frankfurter Buchhändler und Verleger trug damit sowohl der zunehmenden Nachfrage nach Reiseliteratur als auch dem anhaltenden, genuin christlichen Interesse am Heiligen Land Rechnung. Er schlug sozusagen zwei Fliegen mit einer Klappe, als er Pilgerberichte des 13. bis 16. Jahrhunderts mit etwas Kreuzzugsgeschichte (Robertus Monachus), den (fingierten) „Reisen“ des Ritters Johann von Mandeville (in der Bearbeitung Ottos von Diemeringen) und den Explorationen des Augsburger Arztes und Botanikers Leonhard Rauwolf (1573-1576) zwischen zwei Buchdeckeln zusammenschloss. Die Vorrede ließ keinen Zweifel daran, dass vor allem das allgemeine Interesse an Unterhaltung, aber auch Wissbegierde und das Verlangen nach geistlicher Unterrichtung angesprochen werden sollten ${ }^{31}$. Mehrere Auflagen, bis weit ins 17. Jahrhundert hinein, bezeugen, dass der Verleger richtig kalkuliert hatte ${ }^{32}$.

Der Vergleich der handschriftlichen Überlieferung mit dem Druck zeigt, dass Albrechts Reisebericht nur geringfügigen sprachlichen Eingriffen unterzogen wurde, dass aber längere lateinische Passagen (über das Zeremoniell beim Ritterschlag am Heiligen Grab, Pilgerzeugnisse, ein Verzeichnis der heiligen Stätten) ins Deutsche übersetzt wurden und ein kulturgeschichtlich aufschlussreicher Absatz über den Kompass, einschließlich einer Windrose, gänzlich fortfiel. Das eine hätte den Leser mit Dokumenten im originalen Wortlaut konfrontiert, das andere hätte ihn über die Möglichkeiten und Mittel der mediterranen Seefahrt informiert. Doch beides war einer breiteren Leserschaft offenbar nicht zuzumuten. Das wenigstens meinte der Verleger.

Zwei der drei erhaltenen Widmungsexemplare (in Stuttgart und Karlsruhe) zeichnen sich durch ihre vornehme Ausstattung aus. Sie sind in braunes Leder gebunden, Vorder- und Rückseite mit Streicheisenlinien verziert. Mit grünen Seidenbändern ließen sich die Handschriften verschließen. Hinten genügte das bloße Ornament, doch vorne prangt das Wappen des Autors, beides mit Plattenstempel geprägt. Reste der Vergoldung kann man noch erkennen. Die Umschrift: IHERVSALEMISCH RAISBVCH DEO FIDO kündigt den Inhalt des Buchs an. Das Wappen selbst besteht aus vier Feldern mit zwei aufrecht stehenden und zwei schreitenden Löwen, die die Grafschaft Löwenstein und die Herrschaft Scharfeneck repräsentieren. Die Wittelsbacher Rauten auf dem Herzschild in der Mitte weisen auf Albrechts Abstammung von Kurfürst Friedrich dem Siegreichen hin ${ }^{33}$. Über das Wappen wurde das ganze Rad der heiligen Katharina platziert, mitsamt

30 Vgl. Ehmer (wie Anm. 1) S. 170, Anm. 51.

31 Feyerabend (wie Anm.7) fol. II-V.

32 Vgl. Anne Simon, Sigmund Feyerabend's Das Reißbuch deß heyligen Lands (Wissensliteratur im Mittelalter, Bd.32), Wiesbaden 1998.

33 Zum Wappen vgl. Ehmer (wie Anm. 1) S.156f. 
dem Schwert, mit dem die Jungfrau enthauptet, und den spitzen Messern, mit denen sie malträtiert worden sein soll. An einer in der Helmzier befestigten Kette hängt das Jerusalemkreuz, bestehend aus einem Krücken- und vier griechischen Kreuzen, die als Hinweise auf die fünf Wunden Jesu Christi verstanden wurden.

Albrecht von Löwenstein hatte sich am Heiligen Grab zum Ritter schlagen lassen und anschließend auf dem Sinai das Katharinenkloster mit den Reliquien der Heiligen besucht. Hätte er auf die gefahrvolle Weiterreise verzichtet und sich mit Katharinas angeblichem Geburtsort Constantia (Salamis) auf Zypern begnügt, dann hätte er nur das halbe Rad als sein Zeichen beanspruchen können. So aber durfte er sich als Grabes- und Katharinenritter verstehen und beide Zeichen führen, das Kreuz an einer goldenen Kette, wie sie von Albrechts Wappen herabhängt ${ }^{34}$.

Ein Schriftband über dem Schild zeigt seine Initialen: $A$ (lbrecht) $G($ raf $) Z(u) L(e-$ wenstein) $H($ err $) Z(u) S$ (charfen) $E(c k)$. Das Wappen bringt seinen ererbten Status zum Ausdruck, verbessert und vermehrt um die Zeichen seiner persönlichen Verdienste. Denn mit seiner Reise ins Heilige Land und zum Sinai hatte er - zumal unter den erschwerten Bedingungen seiner Epoche - besondere Ehre, nämlich den Rang eines miles Christianus, eines miles sancti sepulchri gewonnen. Indem er Abschriften seines Reiseberichts mit den unterwegs erworbenen Zeichen versah und diese an ausgewählte Standespersonen verschickte, beanspruchte er - ungeachtet aller ostentativen Bescheidenheit in der Vorrede - Respekt und Anerkennung für diese Leistung.

Besondere Aufmerksamkeit kommt der Handschrift Weimar Q 318 zu. Sie ähnelt den drei Widmungsexemplaren, und doch unterscheidet sie sich signifikant von diesen. Sie hat das gleiche Format und einen ähnlichen Einband mit demselben anspruchsvollen Wappen. Hier ist es sogar am besten erhalten (Abb.2). Nur der Buchtitel lautet anders: REISBVCH GEIN IERVSALEM VND VFN BERG SINAI, und die Handschrift wurde offenbar an niemanden verschickt, sondern blieb bei ihrem ersten Besitzer, dem Grafen Albrecht von Löwenstein. Noch 1635 befand sie sich am Ort (oder um genau zu sein: im benachbarten Ottmarsheim), freilich in

34 Vgl. dazu Valmar Cramer, Das Rittertum vom Heiligen Grabe im 14. und 15. Jahrhundert, in: Das Heilige Land in Vergangenheit und Gegenwart 3 (1941) S.111-200, besonders S. 144 ff.; DERs., Das Rittertum vom Hl. Grabe im 16. Jahrhundert, in: Das Heilige Land in Vergangenheit und Gegenwart 4 (1949) S. 81-159, besonders S.109ff.; DERs., Der Ritterorden vom Hl. Grabe von den Kreuzzügen bis zur Gegenwart, Köln ${ }^{2} 1983$, S. 32 ff., 54 ff.; zu Katharina, ihrer Verehrung und zum Sinai: Rudolf Hiestand, Der Sinai - Tor zu anderen Welten, in: Peter Wunderli (Hg.), Reisen in reale und mythische Ferne. Reiseliteratur in Mittelalter und Renaissance (Studia humaniora, Bd.22), Düsseldorf 1993, S. 76-102; Folker Reichert, Wallfahrt zu Gott: Der Sinai als Pilgerziel, in: Helmut BrallTuchel (Hg.), Wallfahrt und Kulturbegegnung. Das Rheinland als Ausgangspunkt und Ziel spätmittelalterlicher Pilgerreisen (Schriften des Heimatvereins der Erkelenzer Lande, Bd.26), Erkelenz 2012, S. 172-187; Anne Simon, The Cult of Saint Katherine of Alexandria in Late-Medieval Nuremberg. Saint and the City, Farnham 2012 (S. 8 f., 51 f. zu Zypern). 
fremdem Besitz ${ }^{35}$. Sie enthält daher keine Widmungsvorrede, ist aber von einer einzigen Hand geschrieben und bietet denselben Text wie die drei Abschriften. Wahrscheinlich handelt es sich um ein Autograph, wahrscheinlich handelt es sich um: das originall ${ }^{36}$.

\section{Ehre und Ruhm}

Aus Ehrerwerb folgt Ehrdemonstration. Das eine gibt dem anderen seinen Sinn. Das Bemühen, den Erfolg der Wallfahrt sichtbar zu machen, gehörte daher zu den Begleit- und Folgeerscheinungen der Heiliglandfahrten. So gesehen, war die Rückkehr wichtiger als die Reise selbst ${ }^{37}$. Gerade die adligen Pilger betrachteten die Wallfahrt nicht nur als geistliches Unternehmen, mit dem sich möglichst viele Ablässe, sondern auch als ritterliches Abenteuer, mit dem sich Ruhm und Ehre gewinnen ließen. Die Reise nach Jerusalem war nämlich mit so vielen Gefahren und Entbehrungen verbunden, dass sie als Mutprobe verstanden werden konnte. Wer sie heil überstand, hatte sich mannhaft bewährt und die Ritterwürde redlich verdient. Jeder Adlige hatte ein Interesse daran, seine Leistung zu dokumentieren und diese seiner Mit- und Nachwelt in möglichst einprägsamer Form vor Augen zu führen. Weniger die eigene Erinnerung zu pflegen als fremde Memoria anzuregen, wurde damit erstrebt ${ }^{38}$.

Verschiedene Möglichkeiten boten sich an. Schon unterwegs (in Pilgerherbergen auf Zypern oder Kreta, auch in bestimmten Kirchen), erst recht am Ziel (in Jerusalem oder im Katharinenkloster auf dem Sinai) hängten adlige Pilger ihre Wappenschilde in Pergament auf und wollten damit zeigen, dass sie hier waren. Auch Graffiti auf Türen, Tischen und Wänden erfüllten diesen Zweck ${ }^{39}$. Zwar war das eine nicht dauerhaft, das andere nicht auffällig. Aber wir besitzen genügend Zeugnisse von Reisenden, die aufmerksam registrierten, wer vor ihnen da war. Gerade Adlige hatten dafür viel Sinn. Albrecht von Löwenstein identifizierte in Candia (Iráklion auf Kreta) 23 Wappen, davon sechs, die auf der Pilgerfahrt des Grafen von Württemberg, Eberhards im Bart, fast hundert Jahre zuvor angebracht worden

${ }^{35}$ Besitzvermerk fol. $132^{\mathrm{r}}$ : Ich Elias Heüßner von vnd zuo Othmarßheim. Von ihm stammen einige Notate.

${ }^{36}$ Unveröffentlichte Beschreibung von Franzjosef Pensel (Arbeitsmittel der AnnaAmalia-Bibliothek).

37 Vgl. Werner Paravicini, Der Grand Tour in der europäischen Geschichte: Zusammenfassung, in: DERs., Grand Tour (wie Anm.15) S.657-674, hier S. 659.

38 Zur „Reise-Memoria“ und zu ihren Funktionen vgl. Treue, Abenteuer (wie Anm.4) S. $289 \mathrm{ff}$.

39 Detlev KraAck, Monumentale Zeugnisse der spätmittelalterlichen Adelsreise. Inschriften und Graffiti des 14.-16. Jahrhunderts (Abhandlungen der Akademie der Wissenschaften zu Göttingen, Phil.-hist. Kl. III, Bd.224), Göttingen 1997. 
waren $^{40}$. Jacob Wormbser fand auf dem Sinai den Namen eines Verwandten und weitere, die er kannte ${ }^{41}$.

Nach der Heimkehr ließen vornehme Pilger wertvolle Teppiche, Gedenktafeln oder Glasfenster herstellen, auf denen sie sich als ruhmreiche Heiliglandpilger inszenierten. Indem sie ihre Mitbringsel, also Reliquien, Devotionalien und Souvenirs, vorzeigten und das eine oder andere sogar herschenkten, ließen sie nicht nur Freunde und Verwandte an ihren Erlebnissen teilhaben, sondern machten gleichzeitig und nachdrücklich auf ihre Verdienste aufmerksam. Die Zeichen der Grabes-, weniger der Katharinenritterschaft, trug man bei festlichen Anlässen, und sie waren geeignet, ein ritterliches Epitaph besonders augenfällig zu schmücken und so den Ruhm ihres Trägers auch über dessen Tod hinaus zu verkünden. Patrizische Familien wie die Nürnberger Ketzel oder Tucher waren an all dem nicht weniger interessiert als der ritterliche Adel oder Fürsten wie Friedrich der Weise von Sachsen und Ottheinrich von der Pfalz ${ }^{42}$. Eine systematische Bestandsaufnahme würde das zeigen.

Fast alles davon wurde auch von Albrecht und seinen Reisegefährten praktiziert. Der Graf selbst hinterließ seinen Namen im Pilgerhospiz in Ramla (Palästina), wo er noch 18 Jahre später gelesen werden konnte ${ }^{43}$. Bartlmä Khevenhüller verewigte sich in Nikosia auf Zypern ${ }^{44}$. Jacob Wormbser brachte von seiner Reise ein Straußenei mit nach Hause. Er ließ es wertvoll einfassen und mit seinem Wappen verzieren. Dann schenkte er es an das Benediktinerinnenkloster St. Johann bei Zabern im Elsass (von wo es irgendwann in die Kunstkammer der Herzöge von Württemberg gelangte). Eine ausführliche Inschrift auf der Fassung erinnert an den Stifter ${ }^{45}$. Die

${ }^{40}$ Folker Reichert, Nachrichten aus Candia und Ramla, in: Gerhard Faix/Folker Reichert, Eberhard im Bart und die Wallfahrt nach Jerusalem im späten Mittelalter (Lebendige Vergangenheit, Bd.20), Stuttgart 1998, S.204-207.

41 KrAACK (wie Anm. 39) S. 444.

42 Vgl. Friedrich H. Hofmann, Denkmäler des Ordens vom hl. Grab in Bayern, in: Das Bayerland 23 (1912) S. 646-651, 661-670; DERs., Wallfahrtsbilder vom heiligen Land, in: Der Kunstwanderer 9 (1927) S.137-139; Theodor Aign, Die Ketzel. Ein Nürnberger Handelsherren- und Jerusalempilgergeschlecht, Neustadt a.d. Aisch 1961; ReIcherT, Die Reise des Pfalzgrafen Ottheinrich (wie Anm.3) S. 58 ff.; Ders., Ehre durch Demut. Wallfahrten des Adels im späten Mittelalter, in: Horst CARL/Sönke Lorenz (Hg.), Gelungene Anpassung? Adelige Antworten auf gesellschaftliche Wandlungsvorgänge vom 14. bis zum 16. Jahrhundert (Schriften zur südwestdeutschen Landeskunde, Bd.53), Ostfildern 2005, S. 165-183 (Nachdruck in: DERs., Asien und Europa im Mittelalter. Studien zur Geschichte des Reisens, Göttingen 2014, S.143-160); Reliquien: DERs., Ein cleins ringlein, an allen heilgen stetten angerürt. Zur materiellen Überlieferung der Heiliglandfahrten im 15. Jahrhundert, in: DA 67 (2011) S. 609-623; Friedrich der Weise: RöHRICHT, Deutsche Pilgerreisen (wie Anm.6) S. 175.

43 Kraack (wie Anm. 39) S. 450; Reichert, Nachrichten (wie Anm. 40) S. 206.

44 KraAcK (wie Anm. 39) S. 445.

45 ANO DOMNI 1562 HATT DIISSES STROVSSEN AIG GEBROCHT VON IHERVSSALEM IACOB WVRMSER DER ELTER VND GESCHENCK DER ERWIRDIGEN FRAWE AMELLEIGEN V. OBERKIRCH EPTISSEN ZV SANNT IOHAN BEII 
Nonnen konnten mit dem Geschenk etwas anfangen. Denn Straußeneier wurden wegen der Eigentümlichkeiten des Vogels - dass er vor dem Legen der Eier angeblich den Himmel anschaut und diese dann auch nicht selbst ausbrütet - entweder als Meditationsobjekte verwendet oder als Sinnbilder der Jungfrauengeburt betrachtet. Am einfachsten konnte man die Eier auf Rhodos erwerben ${ }^{46}$. Allerdings konnte man dort auch sehen, dass nicht alles wirklich zutraf, was man sich von dem wundersamen, angeblich sogar eisenfressenden Tier erzählte ${ }^{47}$.

Jacob Wormbser hielt seinen Namen durch ein großzügiges Geschenk in Erinnerung. Den drei anderen Autoren wurden Grabmäler errichtet, die die Zeichen der Pilgerschaft trugen. Allerdings hatte keines von ihnen ununterbrochen und auf Dauer Bestand. Denn zur Geschichte gehört das Vergessen. Für David Furtenbach gilt das vor allem. In Gaza trennte er sich von seiner Gruppe, um nicht über Kairo, sondern auf direktem Weg zum Sinai zu ziehen. Doch unterwegs erkrankte er an der Roten Ruhr, kam völlig geschwächt im Katharinenkloster an und starb, bevor er die Reliquien der Heiligen verehren konnte. Er wurde im Garten des Klosters beerdigt, aber die Katharinenritterschaft blieb ihm versagt. Seine Mitpilger ritzten in einen großen Stein das Jerusalemkreuz ein und hängten ein provisorisches Epitaph in die Kirche ${ }^{48}$. Nichts davon blieb erhalten.

Auch Bartlmä Khevenhüller durfte das ganze Katharinenrad nicht führen, weil er den Sinai nicht besucht hatte, und das halbe ließ er sich entgehen, als er sich auf Zypern für mancherlei, aber nicht für die Gedenkstätten in Constantia nahe Famagusta interessierte (vielleicht weil er schwer erkrankt war) ${ }^{49}$. Doch mit dem Jerusalemkreuz zeigte er sich offenbar umso lieber. Er wurde 74 Jahre alt, heiratete dreimal und wurde in der Chronik seines Hauses, der Khevenhüller-Chronik, jedes Mal zusammen mit seiner neuen Ehefrau dargestellt, jeweils mit dem Zeichen

ZAWERENN EZ. Vgl. Badisches Landesmuseum Karlsruhe, Die Renaissance im deutschen Südwesten, Karlsruhe 1986, Bd.2, S.639; Sebastian Bock, Ova struthionis. Die Straußeneiobjekte in den Schatz-, Silber- und Kunstkammern Europas, Freiburg i. Br. 2005, S.37, 235.

${ }^{46}$ Johannes TRIPPs, Pilgerfahrten als kreative Impulse für die Goldschmiedekunst der Spätgotik: Stiftungen von Pilger- und Reiseandenken durch Adel und Stadtpatriziat in Kirchenschätze, in: Paravicini, Grand Tour (wie Anm. 15) S.173-190, hier S. 180 ff.

47 E. von Groote (Hg.), Die Pilgerfahrt des Ritters Arnold von Harff ... in den Jahren 1496 bis 1499, Köln 1860, S.71 f.; Helmut Brall-Tuchel/Folker Reichert (Hg.), Rom Jerusalem - Santiago. Das Pilgertagebuch des Ritters Arnold von Harff (1496-1498), Köln ${ }^{3} 2009$, S. 99 f.; Felix Fabri, Evagatorium in Terrae Sanctae, Arabiae et Egypti peregrinationem, hg. von Konrad Dieterich Hassler, Bd.1-3 (Bibliothek des Litterarischen Vereins in Stuttgart 2-4), Stuttgart 1843-1849, Bd.3, S. 27 f.; DERs., Galeere und Karawane. Pilgerreise ins Heilige Land, zum Sinai und nach Ägypten 1483, bearb. von Herbert WiEgAndT, Stuttgart 1996, S.224f.

48 Wormbser, in: Feyerabend (wie Anm.7) fol. 225ㄴ -226v. Vgl. Kraack (wie Anm. 39) S. 444 .

49 Khull (wie Anm. 7) S. 149 ff. 
der Wallfahrt an goldener Kette ${ }^{50}$ (Abb. 3). Er trug damit zum Ruhm seiner Familie bei, die aus bürgerlichen Anfängen zu den höchsten Landesämtern emporgestiegen und zunächst im stadtherrlich-fürstlichen Dienst, dann durch gezielte Heiraten zu Besitz und Ansehen gekommen war ${ }^{51}$. Gleich nach seiner Heimkehr wurden Gerüchte gestreut, die die adlige Abkunft der Khevenhüller in Frage stellen sollten. Die Familie reagierte mit der Anfertigung eines Stammbaums voller genealogischer Erfindungen, und Bartlmä unterstrich mit dem Zeichen der Grabesritterschaft seinen Anspruch auf Gleichrangigkeit mit dem alteingesessenen Adel. Als er im Jahr 1613 verstarb, gab die Witwe bei dem aus Oberitalien stammenden, aber in Klagenfurt tätigen landständischen Polier und Bildhauer Martin Pacobello den Grabstein in Auftrag. Er zeigt das mehrfach gebesserte Khevenhüller'sche Wappen mit seinen vielfältigen Bestandteilen, erläutert durch zwei Schriftbänder mit je einem (links wörtlichen, rechts unwörtlichen) Zitat aus Martin Luthers Bibelübersetzung und ergänzt durch ein geradezu monumentales Jerusalemkreuz, das auch dem Protestanten Bartlmä Khevenhüller etwas wert war ${ }^{52}$. Obwohl er die Wallfahrt ohne die Genehmigung seines älteren Bruders unternommen hatte, wurde das Andenken des Jerusalemfahrers in der familiären Erinnerung weiter gepflegt. Das Grab befand sich in der Kapelle der von Bartlmä zur Prunkburg ausgebauten Feste Landskron bei Villach in Kärnten und wurde wie diese im 19. Jahrhundert dem Verfall preisgegeben ${ }^{53}$. Erst 1955 wurde die Grabplatte bei Restaurierungsarbeiten wieder aufgefunden und aufrecht neben den Eingang zur Kapelle platziert. Da sie aus hartem Pörtschacher Marmor besteht, hat sie die Jahrhunderte weitgehend unbeschädigt überstanden (Abb.4).

50 Karl Dinklage, Kärnten um 1620. Die Bilder der Khevenhüller-Chronik, Wien 1980, S.118, 123, 126. Ebenso Franz Khevenhüller, der auf dem Heimweg verstorben war (ebd., S. 217).

51 Hierzu und zum Folgenden vgl. Wilhelm Neumann, Zur Frühgeschichte der Khevenhüller, in: Neues aus Alt-Villach 15 (1978) S. 61 -84; 16 (1979) S.7-37; Ulrike Öтть, Die Familie Khevenhüller, in: Elisabeth Vavra (Hg.), Die Familie. Ideal und Realität, Horn 1993, S. 335-349; Das Khevenhüller-Archiv. Die Rückkehr eines kulturgeschichtlichen Schatzes, bearb. von Wilhelm WADL, Klagenfurt 2004.

52 Günter Hermann Neckheim, Der Bildhauer Martin Pacobello. Ein Beitrag zur Kunstgeschichte Kärntens im Frühbarock, in: Carinthia I 147 (1957) S.594-619, hier S. 605 f., 616; Richard Milesi, Barock und Klassizismus in der Grabplastik Kärntens (Buchreihe des Landesmuseums für Kärnten, Bd. 18), Klagenfurt 1965, S. 15 ff., Abb. 16 (ohne das Jerusalemkreuz!); Dehio-Handbuch der Kunstdenkmäler Österreichs: Kärnten, bearb. von Gabriele Russwurm-Biró, Wien ${ }^{3} 2001$, S. 442 f.

${ }^{53} \mathrm{Zu}$ Landskron vgl. Hermann WIESSNER, Burgen und Schlösser um Hermagor, Spittal, Villach (Kärntens Burgen und Schlösser, Bd.3), Wien 1967, S.118ff.; Franz X. KoHLA, Kärntens Burgen, Schlösser, Ansitze und wehrhafte Stätten. Ein Beitrag zur Siedlungstopographie. Mit Ergänzungen, Exkursen und Nachträgen von Gotbert Moro (Aus Forschung und Kunst, Bd.17 I), Klagenfurt ${ }^{2} 1973$. 


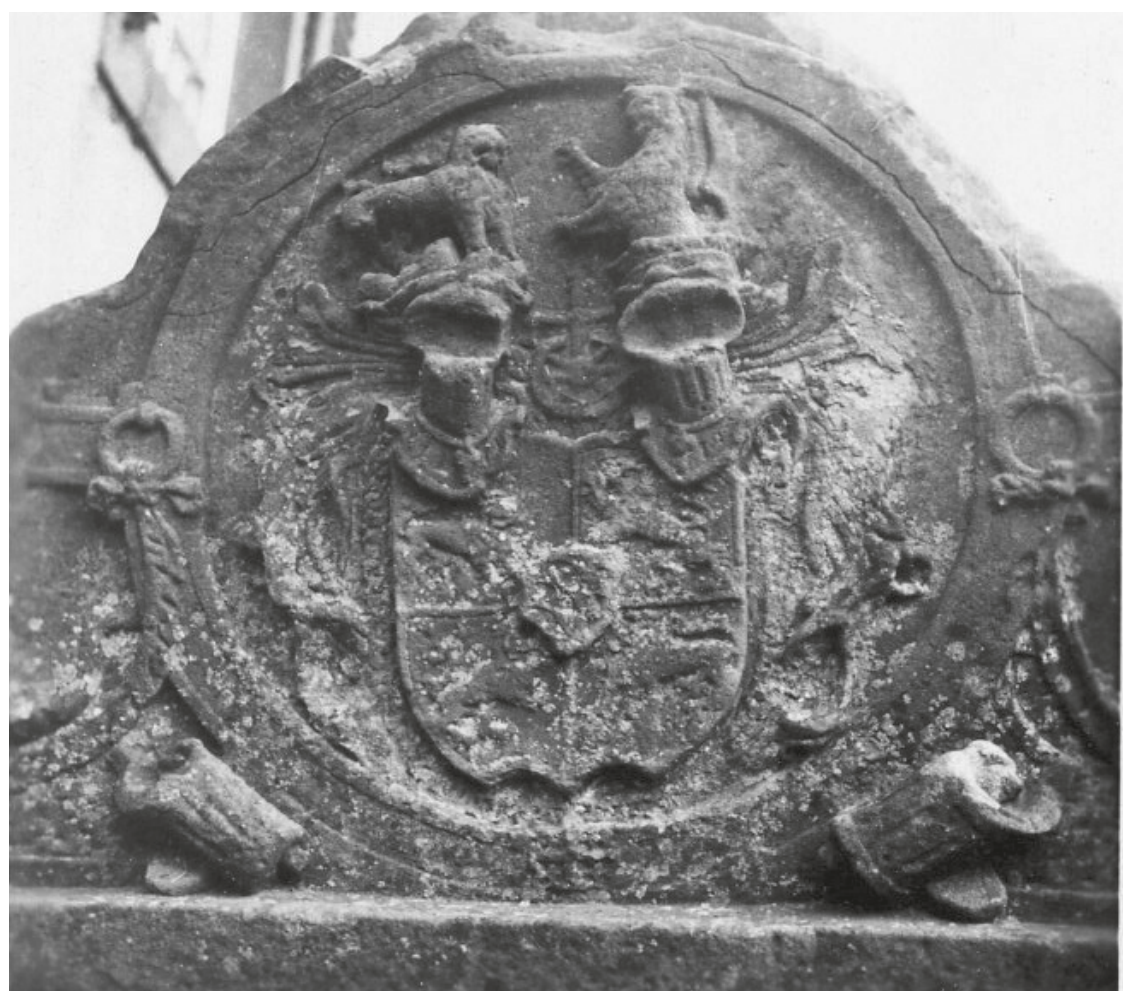

Abb.1: Wappen Albrechts von Löwenstein in Abstatt, Zustand 1951 (StA Wertheim, A 57 I Nr. 119).

Etwas, aber nicht viel besser erging es dem Grabmal, das Albrecht von Löwenstein im Kloster Schöntal erhielt ${ }^{54}$. Wahrscheinlich wurde es 1766 von dort entfernt und nach Abstatt verbracht. Im Freien aufgestellt, war es über zwei Jahrhunderte hinweg den Unbilden der Witterung ausgesetzt. Der Zahn der Zeit nagte an dem Sandstein, und mit den äußeren Konturen ging auch die Erinnerung an den Dargestellten dahin. Über die Identität des „Abstatter Ritters“ wurden Vermutungen geäußert. Erst 1979 erhielt das Grabmal, aus dem ein Denkmal geworden war, einen einigermaßen gesicherten Platz, und Hermann Ehmer gab dem Namenlosen seinen Namen zurück. Die Deutung des gräflichen Wappens über dem Kopf der Figur (Abb.1) spielte dabei die ausschlaggebende Rolle. Dass es durch weitere Zeichen ergänzt wurde, die an Albrechts Heiliglandreise erinnern, war nicht erheblich. Sie sind auch kaum zu erkennen. Wahrscheinlich bemerkt man sie nur,

54 Zum Folgenden Ehmer (wie Anm. 1) S.153ff., $221 \mathrm{f}$. 
wenn man mit ihnen rechnet. Unten hängt stark verwittert das Jerusalemkreuz an langer Kette (Abb.5); etwas deutlicher ragt das Katharinenrad mit Schwert und scharfen Messern aus der Helmzier hervor (Abb. 6). Mit anderen Worten: Es ist das gleiche, um die Zeichen der Pilgerfahrt erweiterte Wappen, das sich auf dem originall von Albrechts Pilgerbericht und ebenso auf den von ihm verschickten Abschriften befindet. Darauf legte er Wert. Man kann sich sogar vorstellen, dass er die Gestaltung seines Grabmals selbst initiiert hat. Es zeigt sich, welche Bedeutung die abenteuerliche und entbehrungsreiche, aber gerade dadurch so verdienstliche Reise nach Jerusalem auch im späten 16. Jahrhundert für das Selbstverständnis des Adels noch besaß.

\section{Anhang}

\section{Albrecht von Löwenstein an Friedrich, Graf von Württemberg und Mömpelgard}

\section{Januar 1580}

\section{Stuttgart, WLB, Cod. hist. qu. 81, fol. $3^{\mathrm{r}}-4^{\mathrm{r}}$ (Widmungsvorrede)}

[Fol.3 r] Dem durchleichtigen hochgebornen fürsten unnd herrn, herrn Früderichen, graven zu Württemberg unnd Monttpelgartt ${ }^{55}$, meinem gnedigen herrn.

Durchleüchtiger hochgeborner fürst! Euren Fürstlichen Gnaden seien, neben wünschung eines newen glickhseligen jars, meine unnderthönige diennst ungeffardts vleis beraitt zuvor, gnediger herr. Durch den auch durchleüchtigen hochgebornen meinen gnedigen fürsten unnd herrn, herczog Ludwigen zu Württenberg ${ }^{56}$, Eurer Fürstlichen Gnaden geliebten herrn vettern, desgleichen auch deren Fürstlicher Gnaden geliebte gemahel ${ }^{57}$, mein gnedige fürstin und frawen, bin ich zu ettlich mallen gnedig angeredt unnd vermanett worden, mein Itinerarium oder Reißbuch über mehr inn das heulig landtt durch Egipten unnd anndere ortt, wie ich dasselb mier uffs kürzest im hin unnd wider raißen zu wasser unnd landtt verzaichnett, in truckh ausgebn zu lasen.

Aber viler bewegender ursachen wegen, die ich Iren Fürstlichen Gnaden eins theils unnderthenig entdeckhett, hab [fol.3v] ich dessen bisher (wie auch noch) bey mier nitt geringes bedennckhens gehabt, ob gleich woll darinnen annders nichts zu finden, dan was ich neben meinen mitgesellen (wölche unnderschidlich hernach benambset werden) mitt augen geseben, auch mitt gefar leibs unnd lebens selbs usstebn und erfaren müessen.

55 Friedrich I., Herzog von Württemberg 1593-1608.

56 Ludwig, Herzog von Württemberg 1568-1593.

57 Dorothea Ursula von Baden-Durlach, seit 1575 mit Herzog Ludwig verheiratet. 
Damitt aber dannocht Ibre Fürstlichen Gnaden, auch hochermeltte derselben gemabel, solcher meiner gannzen rais, auch was mier unnd berürtter meiner gesellschafft in einem unnd den anndern orth begegnett, nichts desto weniger ein volkhommen wissens betten,

So hab ich die selben (wie sie von mier in der hin unnd wider fartt, zu lanndt unnd uff dem mehr mit der kürz verzaichnett) uß dem originall drifach muntiern, die zwey exemplaria ibren beden unnd das dritt Eurer Fürstlichen Gnaden zu anzeigung meines zu derselben habenden guottherzigen gemüetts mitt unnderthenig wünschung eines anfabenden newen glickhselligen jars, teticirn vnnd zustellen wöllen.

Der ungezweiffeltten unnderthönnigen zuver[fol. 4 r] sicht, Eure Fürstliche Gnaden werden solches inn gnaden von mier uff-unnd annemmen, auch hinfüro, wie bisher, mein gnediger herr sein unnd bleibenn, das bin umb Eure Fürstliche Gnaden ich jederzeitt underthönnig zu verdienen willig.

Unnd thue damitt Euren Fürstlichen Gnaden mich jederzeitt zu gnaden underthennig bevelchen, datum am neüen jars tag anno 80

Eurer Fürstlichen Gnaden undertheniger, gehorsammer, williger

Albrecht, graff zu Lewenstein unnd herr zu Scharpffenegckh 


\begin{abstract}
Abb.2: Wappen und Zeichen Albrechts von Löwenstein auf dem Einband seines Reiseberichts (Weimar, Herzogin-Anna-Amalia-Bibliothek, Q 318).

[Die Abbildung kann aus rechtlichen Gründen online nicht bereitgestellt werden.]
\end{abstract}


Abb. 3: Bartlmä Khevenhüller und seine zweite Ehefrau Blanka Ludmilla von Thurn 1593 (aus: Karl DinkLAGE, Kärnten um 1620. Die Bilder der Khevenhüller-Chronik, Wien: Edition Tusch 1980).

[Die Abbildung kann aus rechtlichen Gründen online nicht bereitgestellt werden.]

Zeitschrift für Württembergische Landesgeschichte 75 (2016), S. 97-112

(C) Kommission für geschichtliche Landeskunde in Baden-Württemberg und Württembergischer Geschichts- und Altertumsverein e.V. 


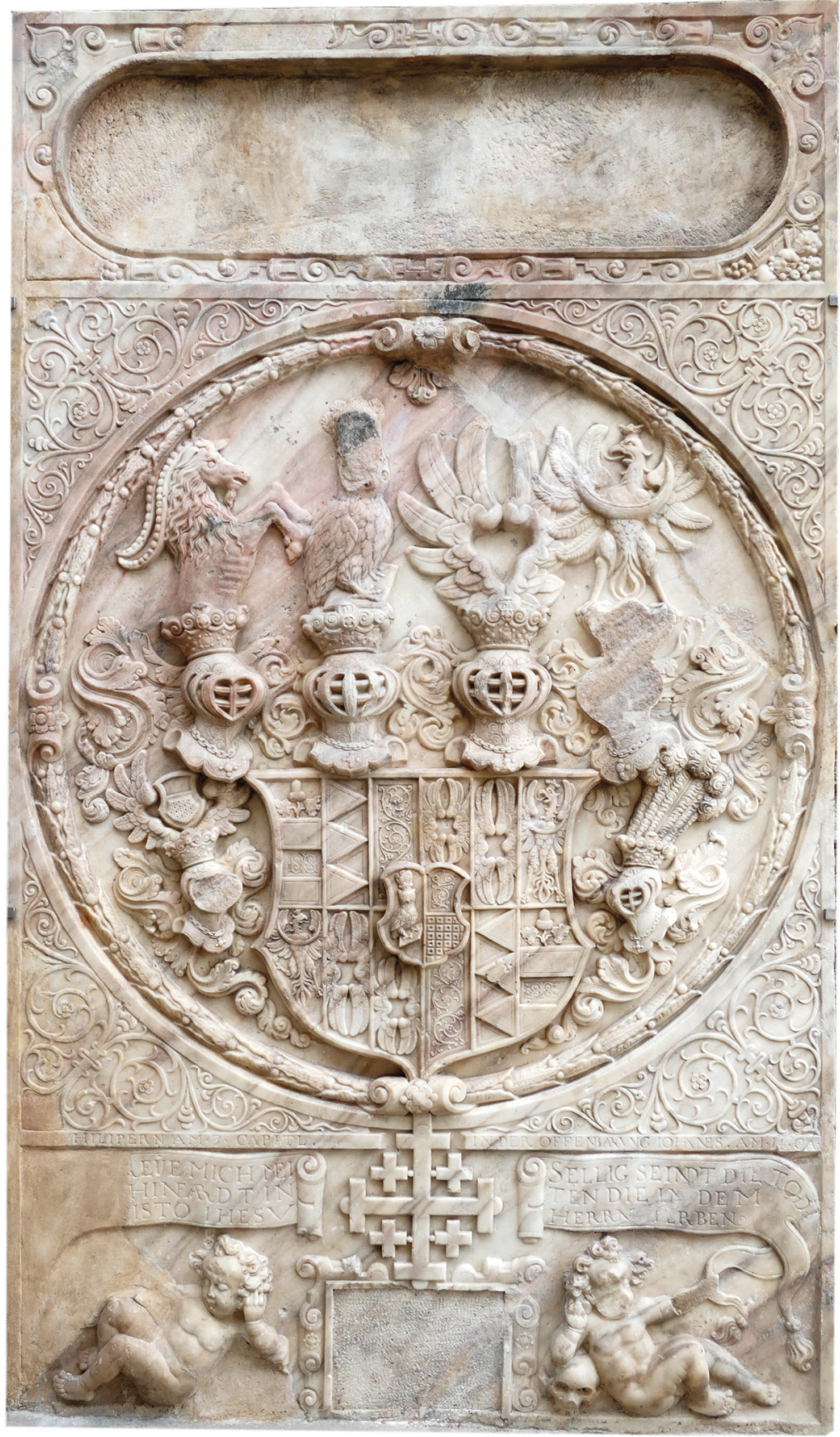

Abb.4: Grabstein Bartlmä Khevenhüllers auf Burg Landskron bei Villach/Kärnten (Foto: Folker Reichert). 




Abb. 5: Jerusalemkreuz unter dem Wappen Albrechts von Löwenstein in Abstatt (vgl. Abb. 1), heutiger Zustand (Foto: Folker Reichert).

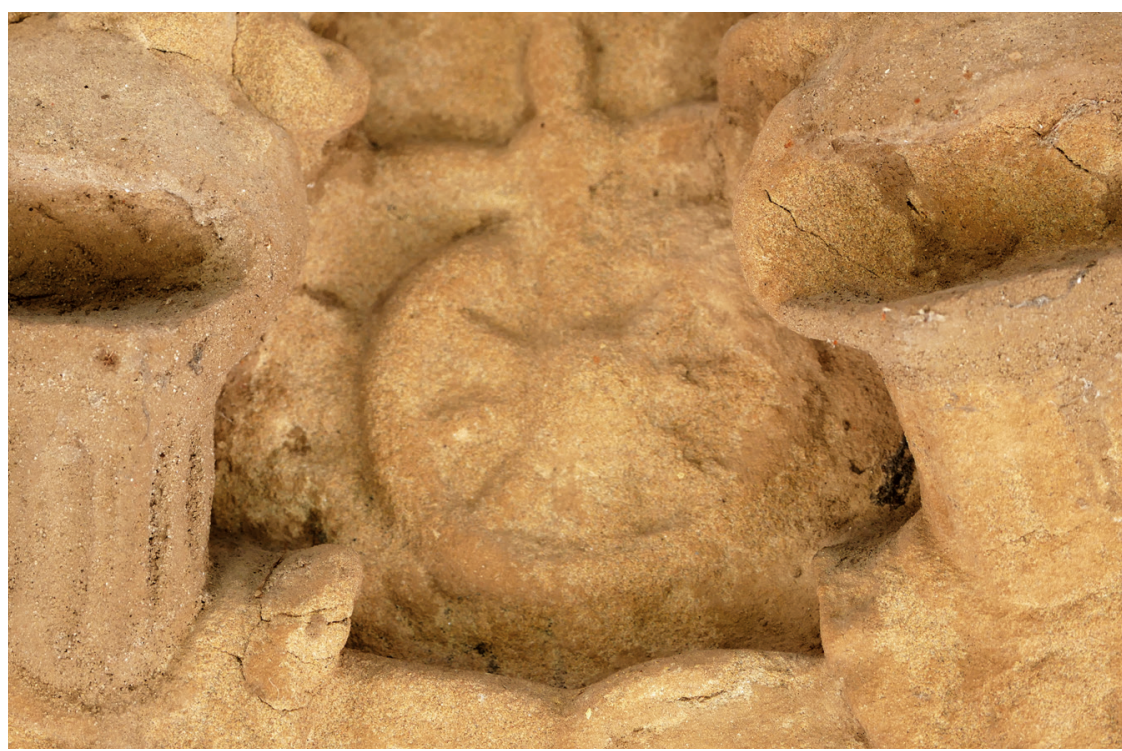

Abb.6: Katharinenrad über dem Wappen Albrechts von Löwenstein in Abstatt (vgl. Abb. 1), heutiger Zustand (Foto: Folker Reichert). 